\title{
EFECTO DEL CULTIVO DE PAPA SOBRE LA FAUNA EDÁFICA EN EL PÁRAMO DE GUERRERO
}

\section{EFFECT OF THE POTATO CROP ON THE SOIL FAUNA AT THE PARAMO GUERRERO}

Liliana H. Casasbuenas Poveda ${ }^{1}$ Luis Hernando Estupiñán Bravo²

\section{RESUMEN}

Las prácticas agrícolas en el páramo alteran la estructura ecológica de las comunidades de la fauna edáfica disminuyendo su composición, su abundancia y su riqueza. El estudio, se desarrolló en el Páramo de Guerrero, Cundinamarca y el objetivo propuesto fue determinar el efecto de prácticas agrícolas sobre la composición de la fauna edáfica. Se muestrearon zonas de páramo sin rastro de intervención antrópica y zonas con presencia de cultivos de papa. La fauna, se obtuvo a partir de monolitos; la extracción e identificación, se efectuó en el laboratorio. Se encontraron 91 morfotipos, siendo los grupos con mayor participación: Arthropoda: Arácnida y Hexapoda. Familias: Entomobryidae, Campodeidae, Staphilinidae, Formicidae, Enicocephalidae y Onychiuridae. Se registraron 695 especimenes, de los cuales el 62,2\% correspondió a la zona sin intervención y el 37,8\% a la zona cultivada. Los mayores valores de abundancia, diversidad y composición, se encontraron en la zona de páramo, que también se caracterizó por la frecuencia de grupos que presentan una baja densidad, que indica la alta especificidad con la disponibilidad de hábitat. En las zonas con cultivos, la frecuencia de la fauna edáfica fue baja al igual que la constancia y la dominancia. El establecimiento de cultivos de papa en el páramo ocasiona cambios en la composición y en la abundancia;

\footnotetext{
${ }^{1}$ Ingeniera Agrónoma. Universidad de Ciencias Aplicadas y Ambientales U.D.C.A lilieca82@yahoo.com.mx. Dirección para correspondencia: Calle 222 No. 54-37. Bogotá.

${ }^{2}$ Biólogo M.Sc. Docente Investigador, Facultad de Ingeniería Agronómica. U.D.C.A luishest@udca.edu.co.
}

algunos grupos desaparecen, mientras que las especies cosmopolitas se adaptan a las condiciones del medio.

Palabras clave: Edafofauna, impacto, páramo, suelo, prácticas agrícolas.

\section{SUMMARY}

Agricultural practices at the Páramo alter the ecological structure of the soil fauna diminishing their composition, abundance and wealth. This study was developed at the Páramo Guerrero, Cundinamarca, with the objective to determine the effect of agricultural practices on the soil fauna. Samples were taken without antropic intervention and in presence of potato crops. The fauna was obtained from monoliths, realizing extraction and identification in the laboratory. 91 morphological types were found, being the groups with more participation: Arthropoda: Aracnida and Hexapoda and Families: Entomobrydae, Campodeidae, Staphilinidae, Formicidae, Enicocephalidae and Onychiuridae. 695 individuals were registered, corresponding $62.2 \%$ to area 1 and $37.8 \%$ to the cultivated area. The highest values of abundance, diversity and composition were in the area of the páramo, characterized also by the frequency of groups that presented a low density, indicating high specificity with the habitat availability. In the areas with cultivations, the frequency of the soil fauna was low, as well as the perseverance and dominance. The establishment of potato cultures in the páramo causes changes in the composition and abundance, some groups disappear, while others, as the cosmopolitan species, increase or adapt to the conditions of the environment.

Key words: Soil fauna, impact, páramo, soil, agricultural practices. 


\section{INTRODUCCIÓN}

Los páramos son considerados ecosistemas de alta fragilidad y sus suelos no son ajenos a esta condición y propensión a procesos de degradación, debido a la baja resistencia y capacidad de recuperación (Alarcón et al. 2002; Gómez, 2002). El uso de los suelos está limitado, por sus características, a una fertilidad natural media a baja, acidez marcada, alta retención de humedad, alta susceptibilidad al deterioro y baja temperatura edáfica que conlleva a una actividad biológica lenta (Cortés, 1995; Malagón E Pulido, 2000). El páramo con el tiempo ha sido transformado en busca de "desarrollo" y de un crecimiento desmedido, se han traspasado sus fronteras y se han intervenido sus recursos. Para Guhl (1982), Gonzáles \& Cárdenas (1995), Vargas-Ríos \& Rivera (1990), Avellaneda (1998), Estupiñán (2001), los factores que están influyendo en la destrucción de los páramos son entre otros: quemas indiscriminadas, sobrepastoreo, establecimiento de plantaciones forestales, introducción de cultivos, utilización intensiva y contaminación del recurso hídrico, extracción de suelos orgánicos y actividades de alto impacto ambiental, como prospección sísmica de hidrocarburos y extracción de minerales. El mayor impacto sobre todos los páramos es, sin lugar a duda, la agricultura, la ganadería y quemas asociadas. Hoy día, un $60 \%$ de todos los páramos están bajo uso continuo y una gran extensión está ocupada con cultivos, pastos sembrados y tierras erosionadas (Hofstede, 2003).

La agricultura genera impactos adversos en los suelos y, por lo tanto, al ecosistema (Alarcón et al. 2002). El suelo es el medio que sostiene y alberga la vida y como tal debe ser considerado como un organismo vivo, que contribuye y hace parte de los diferentes procesos de la naturaleza (Prieto \& Casasbuenas, 2005); además, presenta diferentes grados de sensibilidad a las actividades agropecuarias dependiendo de su naturaleza y su origen (Andrade et al. 2002). El cultivo de papa, tiene beneficios para su desarrollo en los páramos, pero la tecnología utilizada ocasiona erosión, pérdida de la materia orgánica y de su fauna y flora asociada, desequilibrando la dinámica del agua en los suelos y la función del ecosistema como regulador, dejando los suelos inestables, propensos a procesos de reptación, solí fluxión, sofucción, deslizamientos y derrumbes (Alarcón et al. 2002). Se presenta un desequilibrio biológico por la utilización de agroquímicos de manera frecuente y en ocasiones antitécnica, afectando la calidad del suelo y del agua.

Es difícil evaluar y cuantificar los efectos a largo plazo que ocasiona este cultivo, como son desequilibrio en la cadena trófica, acumulación paulatina de tóxicos y la desaparición progresiva de la fauna y microflora del suelo, reguladoras naturales de las poblaciones "plaga" en nichos no perturbados. Estos componentes bióticos han dejado de jugar papeles protagónicos pasando a secundarios y, en los casos más drásticos, han desaparecido (Castaño et al. 2003).

Los organismos que habitan el suelo son importantes para el equilibrio de un ecosistema, por tener efectos positivos en la conservación y en la estructura (Salamanca E Chamorro, 1994; Lavelle, 1990; Lavelle et al. 1993, citados por Morales \& Sarmiento, 2002); actúan en la fragmentación y en la desintegración del material orgánico (Vanegas, 2002; Hoffmann, 1996); intervienen en el incremento de la fertilidad del suelo por la diversidad de nutrientes especialmente del humus [Castillo $\&$ Amat citados por Infante (1987); Cortes, 1995; Parisi, 1979]; actúan en la prevención de la erosión, al proveer una cobertura vegetal y de hojarasca que reduce el impacto de la lluvia sobre la superficie y ayudan a la regulación del clima en el planeta (Varela, 2006).

La degradación del suelo es consecuencia de factores antrópicos ya sea por actuaciones directas, como agrícolas, forestales y de ganadería, o por acciones indirectas, como actividades industriales. Algunos organismos resisten la bioturbación, mientras que en otras ocasiones desaparecen las poblaciones originales y los espacios son ocupados por especies cosmopolitas (Ruiz, 2006). Wall et al. (2004) citados por Varela (2006) afirman que los organismos del suelo están directamente amenazados por las prácticas agrícolas y forestales no sostenibles. Estas prácticas, según Marín et al. (2001), causan alteraciones en la estructura, la composición, la riqueza y la distribución espacial de la fauna edáfica. Coral \& Bonilla (1998) citado por Ramírez et al. (2005) expresan que el cambio del uso de la tierra genera variaciones en las poblaciones como respuesta a modificaciones en la cobertura vegetal, radiación solar, lluvias y propiedades físicas y químicas del suelo. Para Pashanasi (2001), el desmonte y los cultivos sucesivos destruyen rápidamente los macroinvertebrados. En 
áreas con cultivos anuales y bianuales, con relación con los bosques primarios, la biomasa es reducida y desaparecen la mayoría de los organismos. Decaens et al. citados por Ruiz (2006) encontraron que los cultivos anuales tienen un efecto nocivo sobre las poblaciones de lombrices y artrópodos; señalan que los factores responsables de este fenómeno son la utilización de fertilizantes, desaparición del mantillo y modificaciones climáticas que resultan de la tala de vegetación. Estos, junto con la aplicación de plaguicidas, afectan en mayor proporción la diversidad y la abundancia de las comunidades del suelo. Peredo et al. (2002) afirman que altas y repetidas dosis de fertilizantes minerales pueden perjudicar a la mesofauna, ya sea por su toxicidad o por la alta presión osmótica causada; excesos de nitrógeno afectan indirectamente a la mesofauna edáfica al producirse una mayor acidificación del suelo, lo que trae consigo inhibición del crecimiento y actividad microbiana, alterando la fuente de alimentación de la mesofauna. También reportan disminución de la abundancia de microartrópodos edáficos al usar, por ejemplo, glifosato para el control de arvenses, ya que este herbicida, al llegar al suelo provoca efectos sobre la mesofauna. Concluyen, que el efecto prolongado de las labores agrícolas causa una alteración de la estructura del ecosistema representada tanto por una disminución de la abundancia de individuos como de la riqueza. Ramírez et al. (2005) afirman que la disminución o ausencia de la macrofauna en sitios intervenidos es producto del efecto antrópico continuo.

El objetivo general del presente estudio fue determinar el efecto de prácticas agrícolas, relacionadas con el cultivo de papa, sobre la estructura y composición de la fauna edáfica en el Páramo de Guerrero.

\section{MATERIALES Y MÉTODOS}

El Páramo de Guerrero se localiza en la cordillera Oriental, ocupa una área de 15.000 hectáreas que comprende una franja de montaña, a una altitud de 3.000 a $3.900 \mathrm{msnm}$, ubicada entre los $74^{\circ} 10^{\prime} \mathrm{W}$ y los $5^{\circ} 15^{\prime} \mathrm{N}$. El estudio, se desarrolló en el sector de Guargua, entre 3.037 y 3.602msnm, municipio de Carmen de Carupa. El paisaje de la zona, se caracteriza por ser quebrado, de grandes ondulaciones y presentar algunas llanuras y cerros (Alcaldía Municipal, Carmen de Carupa, 2007).
Las zonas donde se realizaron los muestreos fueron las siguientes: Zona 1 (Z1) páramo sin rastro de intervención; Zona 2 (Z2), cultivo de papa en pleno desarrollo; Zona 3 (Z3) cultivo de papa, en la cual finalizaba el ciclo de uno y se iniciaba la siembra de otro y Zona 4 (Z4), el $50 \%$ del tiempo de muestreo permaneció en descanso (barbecho) y en el 50\% restante en cultivo. Se realizaron cuatro muestreos, entre julio y noviembre del 2006.

En cada muestreo, se tomó un monolito de 20x20x20cm, según la metodología propuesta por Anderson $\mathcal{E}$ Ingram (1989). Cada muestra, se dividió en dos: los primeros $10 \mathrm{~cm}$ y los $10 \mathrm{~cm}$ siguientes. La extracción de la edafofauna, se efectuó por el método de BerlesseTullgren (Prieto \& Casasbuenas, 2005). La identificación, se realizó hasta familia, cuando fue posible, utilizando las claves de Borror et al. (1989), Peterson (1960). Se trabajaron morfotipos, que corresponden a la agrupación de organismos de acuerdo a sus características morfológicas compartidas (Wallter E Proctor 2005). Se contó con material identificado de referencia perteneciente a la Colección Biológica de la U.D.C.A (Código 51).

Se determinó riqueza, abundancia poblacional, frecuencia, constancia, dominancia, diversidad (índice de Margalef) y similaridad (índice de Bray Curtis), para establecer la correlación ecológica y determinar el estado de la fauna edáfica en el área de estudio. Se realizó un análisis temporal relacionando valores de precipitación media con los de diversidad a través del tiempo. Los datos climáticos fueron obtenidos de la estación pluviométrica "El Hato" de la CAR. Con la información lograda, se elaboró un análisis multivariado mediante componentes principales (ACP) utilizando programas SAS y SPAD.

\section{RESULTADOS Y DISCUSIÓN}

En la zona 1, la comunidad vegetal dominante es Pajonal - Frailejonal que se constituye como albergue y fuente de alimento para los organismos habitantes del suelo. Para los sitios de muestreo ubicados en las zonas 2, 3 y 4 , se puede apreciar la transformación del páramo natural y las variaciones que tienen las áreas cultivadas con papa de acuerdo a las labores y el uso del suelo. Los cultivos remplazan la vegetación natural y la diversidad vegetal se ve reducida por el establecimiento de un monocultivo 
que usa el suelo como medio de producción y las funciones de la vegetación natural quedan alteradas propiciando que se generen condiciones de hábitat distintas para la fauna edáfica natural, disminuyendo considerablemente la diversidad de organismos del suelo y facilitando el establecimiento de otros que se adaptan a las nuevas condiciones.

Composición de la edafofauna: Se encontraron 91 morfotipos, el Phyllum con mayor participación fue Arthropoda y las Clases más representativas fueron Arácnida y Hexapoda: a nivel de Orden, Acari es el dominante, seguido por Collembolla. Estos dos grupos conforman el 58,0\% de la fauna encontrada, mientras que Coleoptera, otros Hexápodos, Diplura, Psocoptera, Nematoda, Hymenoptera, Geophylomorfa y otros, conforman el valor restante. Las familias que reportaron mayor participación fueron Entomobryidae, larvas de Coleoptera, Campodeidae, larvas de Diptera, Staphylinidae, Formicidae, Enicocephalidae y Onychiuridae (Tabla 1).

El páramo natural (Z1) presentó una composición variada y con representantes de la mayor parte de los grupos taxonómicos encontrados. Predominó Acari, seguido de Entomobryidae, otros Hexápodos, Campodeidae, larvas de Coleoptera y de Diptera, los otros grupos están distribuidos a lo largo de las muestras. Se resalta que de los 28 grupos taxonómicos encontrados para el páramo natural siempre se registraron de 10 a 14 por muestra, lo que indica que existe poca variación en cuanto a composición para dicha zona. De acuerdo con los resultados, se puede afirmar que el páramo suministra las condiciones necesarias para el establecimiento y desarrollo de la biota natural del suelo.

En la zona 2, de la cual se tomaron muestras desde la siembra de la papa hasta que esta cumplió cuatro meses de desarrollo, se pudo apreciar una disminución en la composición de la fauna edáfica; únicamente se encontraron nueve representantes de los 28 grupos taxonómicos, unos pocos se encuentran distribuidos a lo largo de los muestreos. Los grupos más representativos fueron: Acari, presente en todos los muestreos, Collembola, larvas de Coleoptera y Psocoptera, los cuales se encuentran en dos de los cuatro muestreos. Es importante resaltar que la composición disminuye a medida que se desarrolla el cultivo; inicialmente, se hallaron representados 5 y 6 grupos taxonómicos, pero con el tiempo disminuyeron a 3 y 2 , debido a las condiciones dadas por el establecimiento del cultivo y la presencia de precipitaciones altas.

En la zona 3, la composición no varió. Ocho grupos mostraron mayor aporte y fueron los más relevantes en todos los muestreos: Acari, Psocoptera y Entomobryidae; los demás se encontraron en el muestreo dos, pero no se mantuvieron. La escasa composición de esta zona tuvo su origen en el uso del laboreo constante del suelo y otras actividades que agotaron y no permitieron la recuperación de sus características físicas, químicas y/o biológicas.

En la zona 4, se encontró que, a medida que avanzó el desarrollo del cultivo, los grupos disminuyeron, solamente se presentaron nueve, similar a la situación anterior; Acari fue el más representativo. Otros grupos que se encontraron bien distribuidos fueron los coleópteros y los Psocopteros.

La composición de fauna edáfica para las zonas cultivadas con papa, confrontada con la del páramo natural, se vio reducida en un $67,1 \%$, que a lo largo del tiempo presentó marcadas variaciones, indicando que la actividad agrícola tiene una influencia directa negativa sobre la composición de la fauna del suelo.

Riqueza: Se encontraron 28 grupos, en la zona 1, que representan el $96,4 \%$ de los reportados en todos los muestreos. No se encontraron individuos de la Familia Cecidomyiidae que sí estuvieron presentes en la zona 2. Exclusivos para esta zona fueron 15 grupos y 12 compartidos con las zonas 2, 3 y 4 . Acari, Staphylinidae, larvas de Coleoptera, Aphidoidae, Psocoptera y Entomobryidae fueron los más representativos en los muestreos, destacándose la capacidad que probablemente tienen para adaptarse a condiciones extremas, a las cuales se ha llegado por el uso intensivo del suelo por los cultivos de papa (Figura 1). La mayoría de los Staphylinidae encontrados son reconocidos como depredadores. 


\begin{tabular}{|c|c|c|c|c|c|c|c|c|c|c|c|c|c|c|c|c|c|c|c|c|c|c|c|c|c|}
\hline \multirow{4}{*}{ 口 } & $\stackrel{⿱}{N}$ & & $\leq$ & & & & $\underline{=}$ & & $\stackrel{\varrho}{=}$ & $\cong$ & & & & & & 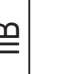 & $\cong$ & $\cong$ & 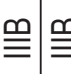 & $\underline{\underline{E}}$ & & & & & \\
\hline & $\stackrel{\sim}{N}$ & & $\leq$ & & & & & & $\stackrel{\varrho}{=}$ & $\cong$ & & & & $\underline{\underline{\underline{m}}}$ & & 包 & $\leq$ & $\cong$ & & & & & $\cong$ & & \\
\hline & $\tilde{N}$ & & $\leq$ & & & $\underline{\underline{\underline{\equiv}}}$ & & & $\stackrel{\varrho}{\equiv}$ & $\cong$ & $\cong$ & & & & & 品 & $\cong$ & $\cong$ & $\cong$ & & & & & & \\
\hline & $\bar{N}$ & $\cong$ & $\leq$ & $\cong$ & $\cong \cong$ & $\cong \cong$ & $\stackrel{\underline{\underline{m}}}{=}$ & $\underline{\underline{\equiv}} \cong$ & $\cong$ & $\cong$ & & $\cong \cong$ & $\underline{\underline{\Xi}} \cong$ & $\underline{\underline{ }} \cong$ & $\cong \stackrel{\varrho}{=}$ & 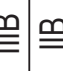 & $\cong$ & $\leq$ & $\cong \cong$ & $\cong \cong$ & 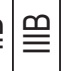 & $\cong$ & $\stackrel{\varrho}{\equiv} \cong$ & $\cong$ & $\leqq$ \\
\hline \multirow{4}{*}{ ত্口 } & N & 0 & 응 & 010 & $\bar{O} 01$ & 0 & 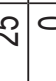 & 010 & 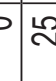 & 2 & 0 & 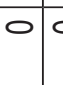 & $\begin{array}{ll}0 & 0 \\
\end{array}$ & 0 & 0.5 & 0 & 品 & ผ & $\stackrel{2}{2}$ & 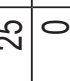 & 0 & 0 & \begin{tabular}{l|l}
0 & 0
\end{tabular} & 0 & 0 \\
\hline & $\stackrel{?}{N}$ & 0 & 음 & $0 \mid c$ & 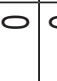 & 015 & 0 & 0. & $\sim \approx$ & in & 0 & 010 & 0 눙 & $\stackrel{\sim}{\sim}$ & 0 눙 & $\begin{array}{lll}N & 0 \\
\end{array}$ & 8 & 10 & 00 & 0 & 0 & 0 & $\stackrel{2}{\sim} 0$ & 0 & 0 \\
\hline & N & 0 & 응 & 010 & 0 't & $\stackrel{\sim}{\sim}$ & $P C$ & 0.0 & $\sim \sim$ & i & $\approx$ & $0 \mid c$ & 010 & 0 & $0 \stackrel{1}{\circ}$ & 20 & in & 员 & 品 & 00 & 0 & 0 & \begin{tabular}{l|l}
0 & 0
\end{tabular} & 0 & 0 \\
\hline & $\bar{N}$ & $\stackrel{2}{2}$ & 음 & $\stackrel{2}{\circ}$ & N2 & 요 & م) & $\stackrel{2}{2}$ & ง. & $\frac{1}{2}$ & 0 & $\stackrel{2}{2}$ & & 8 & เి & $\begin{array}{l}2 \\
N\end{array}$ & 20 & 은 & $\stackrel{2}{2} \stackrel{2}{n}$ & \begin{tabular}{l|l}
$\sim$ & 10 \\
$N$
\end{tabular} & $\stackrel{2}{2}$ & 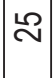 & \begin{tabular}{l|l}
$\stackrel{\sim}{\sim}$ & $\sim$
\end{tabular} & $\stackrel{2}{\sim}$ & $\stackrel{2}{2}$ \\
\hline \multirow{4}{*}{$\frac{4}{\circ}$} & $\stackrel{J}{N}$ & 0 & 응 & \begin{tabular}{l|c}
0 & $C$ \\
\end{tabular} & $\bar{O} 01$ & 0 : & 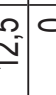 & 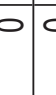 & $\stackrel{n}{\sim}$ & in & 0 & $\begin{array}{lll}0 & \end{array}$ & $\begin{array}{llc}0 & \end{array}$ & $\begin{array}{lll}0 & \\
\end{array}$ & 0 o & No & \begin{tabular}{|l|}
0 \\
0 \\
$m$
\end{tabular} & $\stackrel{0}{\simeq}$ & $\stackrel{2}{\stackrel{0}{\simeq}} \stackrel{5}{7}$ & 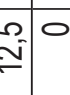 & 0 & 0 & \begin{tabular}{l|l}
0 & 0
\end{tabular} & 0 & 0 \\
\hline & $\stackrel{\sim}{N}$ & 0 & 8 & 010 & $\bar{O} 0.6$ & 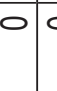 & 50 & 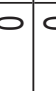 & $\stackrel{0}{\sim}$ & 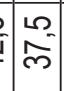 & 0 & 010 & 0.5 & 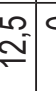 & 0 & 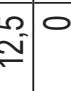 & 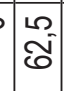 & 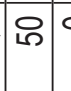 & \begin{tabular}{l|c}
0 & 0
\end{tabular} & \begin{tabular}{l|l}
0 & 0
\end{tabular} & 0 & 0 & \begin{tabular}{|l|l|}
\multirow{2}{*}{} & 0 \\
$\stackrel{1}{\simeq}$ & \\
\end{tabular} & 0 & 0 \\
\hline & N & 0 & \begin{tabular}{|l|}
0 \\
0 \\
0 \\
0
\end{tabular} & 00 & 0 : & 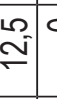 & $8 c$ & 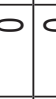 & $\stackrel{2}{\sim}$ & 2 & $\stackrel{\substack{0 \\
\sim}}{\sim}$ & 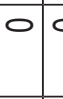 & 010 & 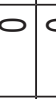 & 0.5 & 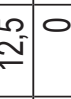 & 2 & $\stackrel{2}{\sim}$ & $\stackrel{2}{\sim}$ & 00 & 0 & 0 & \begin{tabular}{l|l|}
0 & 0
\end{tabular} & 0 & 0 \\
\hline & $\bar{N}$ & 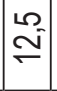 & \begin{tabular}{|l|}
1 \\
0 \\
0 \\
0
\end{tabular} & 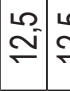 & $\stackrel{2}{\simeq}$ & $\stackrel{2}{2}$ & $\stackrel{2}{2} \stackrel{5}{7}$ & 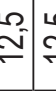 & $\begin{array}{l}0 \\
v \\
\end{array}$ & 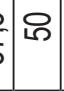 & 0 & $\stackrel{0}{\stackrel{0}{\sim}}$ & $\stackrel{0}{\stackrel{0}{\sim}}$ & 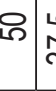 & 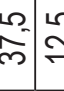 & $\begin{array}{lll}0 \\
\end{array}$ & 5 & $\begin{array}{l}1 \\
0 \\
0 \\
0\end{array}$ & $\stackrel{2}{\stackrel{2}{\simeq}} \stackrel{5}{7}$ & 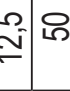 & $\stackrel{\infty}{\sim}$ & $\stackrel{0}{\sim}$ & $\stackrel{2}{\sim} \stackrel{\sim}{\simeq}$ & $\stackrel{0}{\sim}$ & $\begin{array}{l}0 \\
\widetilde{0}\end{array}$ \\
\hline & $\stackrel{\circ}{\text { Oㅇ }}$ & - & $\infty$ & $\sim$ & - & $\nabla c$ & v & - & $-m$ & 으 & - & - & $-c$ & 6. & - & -- & $m$ & $m$ & $\sim-$ & $-\nabla$ & - & - & $-\quad-$ & - & - \\
\hline & $\underset{⿱ 亠 幺}{\stackrel{\leftrightarrows}{\rightleftarrows}}$ & 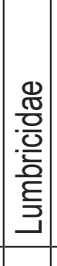 & & & $\begin{array}{ll}0 \\
\frac{0}{0} \\
\frac{0}{0} \\
\frac{0}{0} \\
0\end{array}$ & 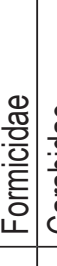 & 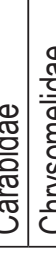 & 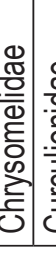 & 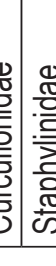 & 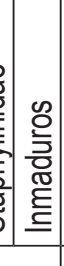 & 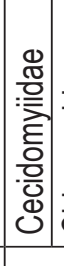 & 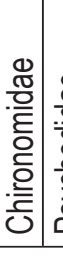 & 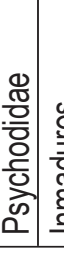 & : & 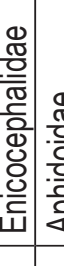 & 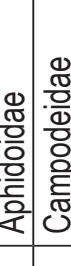 & & 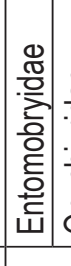 & 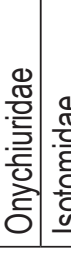 & 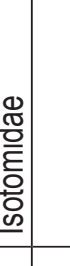 & & 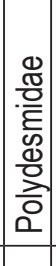 & 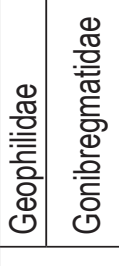 & 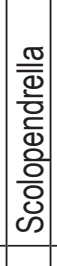 & \\
\hline & $\begin{array}{l}\text { 뼘 } \\
\text { 呂 }\end{array}$ & 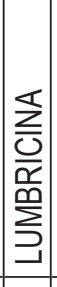 & 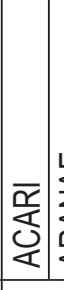 & 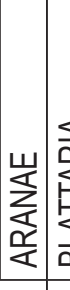 & 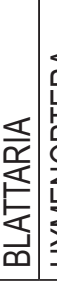 & 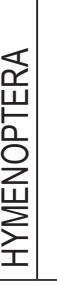 & & c & כ) & & & 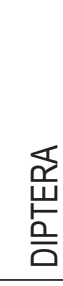 & & & 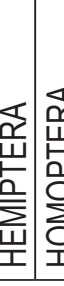 & 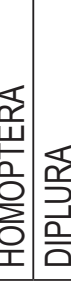 & 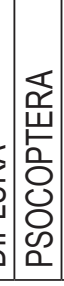 & & 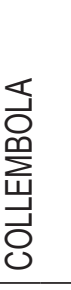 & 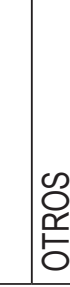 & 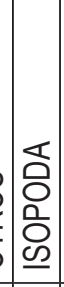 & 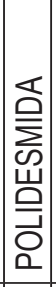 & 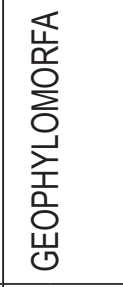 & & \\
\hline & 岁 & & 을 & 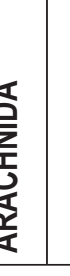 & & & & & & & & & 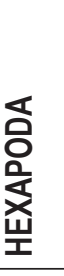 & & & & & & & & 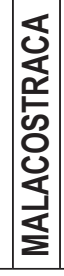 & 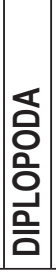 & $\begin{array}{l}\text { 옹 } \\
\text { 응 } \\
\text { 퐁 }\end{array}$ & $\frac{5}{\sum_{\infty}^{1}}$ & \\
\hline & $\begin{array}{l}\text { ఏ } \\
\text { 콤 }\end{array}$ & 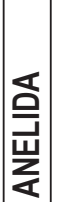 & & & & & & & & & & & & . & 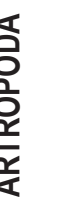 & & & & & & & & & & $\begin{array}{l}\text { 吉 } \\
\text { 일 } \\
\text { 玄 }\end{array}$ \\
\hline
\end{tabular}




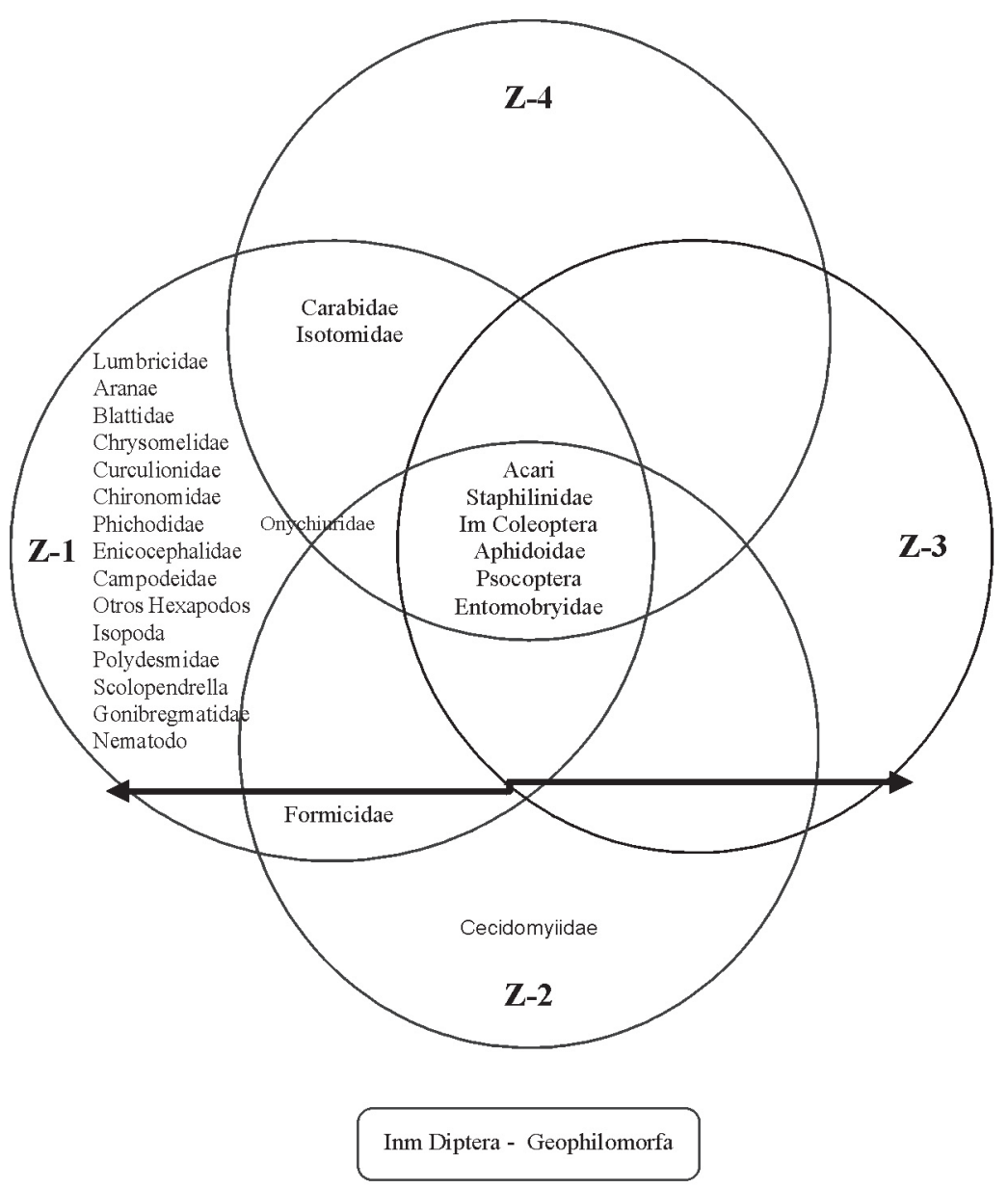

Figura 1. Distribución de los Grupos Taxonómicos en el área de estudio. Z-1, páramo natural sin intervención antrópica; Z-2, corresponde a cultivo de papa en pleno desarrollo; Z-3, cultivo de papa, en la cual finalizaba el ciclo de uno y se iniciaba la siembra de otro y Z-4, zona en la cual el 50\% del tiempo de muestreo permaneció en descanso (barbecho) y el 50\% restante en cultivo.

Los grupos exclusivos para la zona 1 fueron: Lumbricidae, Aranae, Blattidae, Chrysomelidae, Curculionidae, Chyronomidae, Psichodidae, Enicocephalidae, Campodeidae. Otros Hexapodos, Isopoda, Polydesmidae, Scolopendrella, Gonibregmatidae y Nematodo (Figura 1). Posiblemente estos grupos son sensibles a los cambios producidos en el suelo por la actividad agrícola, ya que desaparecen totalmente para las zonas con cultivos.

Se determinaron 91 morfotipos distribuidos a lo largo del estudio. En la zona 1, 69; zona 2, 24; zona 3, 15 y zona 4, 18. Los mayores valores se presentaron, para todas las zonas, en el muestreo dos, así: 37, 11, 9 y 10 morfotipos; los más bajos se registraron en el muestreo 4 con 13, 3, 5 y 5, posiblemente por la presencia de precipitaciones altas que influyeron directamente sobre la riqueza.
Abundancia: Se registraron 695 individuos, 432 (62,2\%) correspondientes a la zona 1 y 263 (37,8\%) al área cultivada. La zona 2 presentó 77 individuos, la zona 3, 71 individuos y la 4: 115 individuos. Los valores alcanzaron su máximo en el páramo natural con marcadas diferencias con las áreas cultivadas (Figura 2). Los valores altos para la zona 1, se debieron probablemente a las condiciones dadas por los recursos de la vegetación correspondientes a la comunidad propia del ecosistema paramuno. Es interesante anotar que Acari, larvas de Coleoptera, Psocoptera y Entomobryidae fueron los grupos más predominantes en la composición; esta situación se ve bien reflejada para la abundancia, ya que estuvieron representados con los mayores valores para todas las zonas.

Los organismos que ocupan un área determinada presentan una distribución de acuerdo con su actividad, 
sus preferencias por la variedad de hábitat, su alimento y la estratificación de la vegetación (Bernal, 1985). Dado que el trabajo se realizó principalmente en áreas cultivadas de papa, se destacó que una de las abundancias totales más altas corresponde a larvas de Coleoptera, que representan una de las principales plagas que afectan los tubérculos.

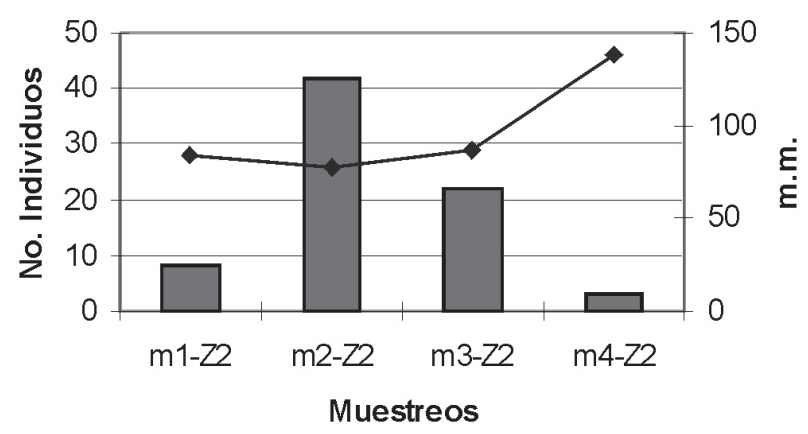

(B)

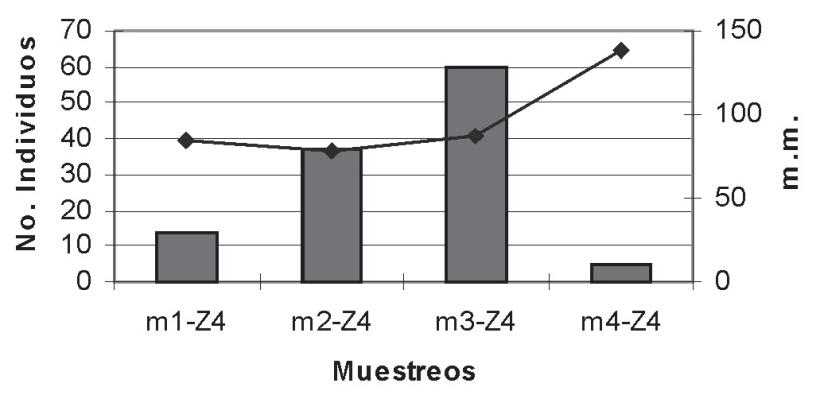

$\square$ No. Individuos $\longrightarrow$ Precipitación

(D)

Figura 2. Variación de la fauna edáfica relacionando la abundancia y la precipitación. $\mathbf{m}$ indica el muestreo y $\mathbf{Z}$, la zona.

Relacionando la abundancia con la precipitación, se encontraron abundancias altas con precipitaciones medias, en el caso del muestreo 2 y las menores abundancias con las mayores precipitaciones, en el muestreo 4 (Figura 2).

Frecuencia, constancia y dominancia: El páramo está caracterizado por la frecuencia de grupos que presentan una baja densidad (Tabla 1), lo que indica la alta especificidad de estos organismos con la disponibilidad de hábitat, algunos de ellos son Lumbricidae, Aranae, Blattidae, Chrysomelidae, Curculionidae, Chyronomidae, Psichodidae, Enicocephalidae, Campodeidae, Isopoda, Polidesmidae. Acari, Entomobryidae y Nematoda que, en la zona 1, ocupan la categoría de muy alta y alta densidad, respectivamente. Los dos primeros, se distinguen por su alta capacidad de adaptación a situaciones adversas del medio que son originadas de forma natural o inducida.

La constancia indica que los grupos Acari, larvas de Diptera y Entomobryidae tienen un comportamiento euconstante. La categoría constante esta representada por inmaduros de Coleoptera, Campodeidae, Pscoptera, otros Hexapodos y Nematoda. Los grupos restantes solamente se presentaron en uno o como máximo dos muestreos, considerándose accidentales. Como resultado de las interacciones de frecuencia y dominancia para los grupos del páramo natural, se clasifican tres grupos dominantes, como superiores Acari y Entomobryidae y como inferior a inmaduros de Diptera. Dentro del rango intermedio superior está solamente Nematoda y en la 
inferior Formicidae, Carabidae, Staphylinidae, inmaduros de Coleoptera, Enicocephalidae, Campodeidae, Psocoptera y otros Hexapodos; los restantes 15 grupos son clasificados como secundarios inferiores.

En la zona 2, solamente Acari tuvo una alta densidad, de los ocho grupos restantes, únicamente Cecidomyiidae es exclusivo para esta zona y, al igual que los demás, presentó una baja densidad. Este mismo comportamiento lo tuvo la constancia donde Acari se resalta, ya que fue el único euconstante, mientras que los demás fueron accidentales o su presencia se considera accidental y, por lo tanto, se le da el calificativo de dominante superior (Tabla 1).

La frecuencia de la zona 3 fue similar que la zona 2, Acari y Psocoptera con muy alta y alta frecuencia en comparación con los seis restantes. Larvas de Díptera y Geophilidae solamente se presentaron en esta zona, debido a su característica de alta movilidad presentada en los individuos adultos. Esto se confirmó por la constancia que presentan estos grupos, los cuales estuvieron en los cuatro muestreos y son considerados como euconstantes. Como constantes, se tuvo a Entomobryidae y el resto se pueden considerar organismos accidentales, donde sobresalieron por su dominancia superior Acari y Psocoptera (Tabla 1).
La zona 4 presentó 9 grupos, 7 en común con las otras zonas de cultivo. Esta zona compartió con páramo sin alterar, Carabidae e Isotomidae. Acari fue el único que mostró muy alta densidad, fue euconstante y su dominancia fue superior frente al resto. A nivel de constancia predominaron los organismos accidentales, específicamente, secundarios inferiores (Tabla 1). A lo largo de los muestreos se evidenció una apreciable disminución de los grupos del páramo frente a aquellas donde el suelo es laboreado, la frecuencia de la fauna edáfica fue baja, al igual que la constancia y la dominancia.

El comportamiento temporal de los organismos del suelo de páramo en las zonas 2, 3 y 4 queda supeditado a las oportunidades que genere el hombre en las actividades del cultivo. Las condiciones del cultivo a nivel de altura, estado fenológico y descanso del terreno fueron diferentes y no cambió el comportamiento en la distribución temporal.

Diversidad: Los mayores valores se encontraron en la zona 1, donde fluctuaron desde 4,88 hasta 3,25. Para las zonas cultivadas, los resultados obtenidos, en comparación a la zona anterior, fueron bajos. En el muestro 2, zona 2, el índice de diversidad fue 2,73 y en el muestreo 1, zona 3, el valor fue 1,52 (Tabla 2). Los

Tabla 2. Abundancia, Riqueza y Diversidad Fauna Edáfica Páramo de Guerrero.

\begin{tabular}{|c|c|c|c|c|c|c|c|c|c|c|c|c|c|c|c|c|}
\hline & \multicolumn{8}{|c|}{ M1 } & \multicolumn{8}{|c|}{ M2 } \\
\hline & \multicolumn{2}{|c|}{$\mathrm{m} 1-\mathrm{Z1}$} & \multicolumn{2}{|c|}{$\mathrm{m1-Z2}$} & \multicolumn{2}{|c|}{ M1-Z3 } & \multicolumn{2}{|c|}{ m1-Z4 } & \multicolumn{2}{|c|}{ m2-Z1 } & \multicolumn{2}{|c|}{$m 2-Z 2$} & \multicolumn{2}{|c|}{$m 2-Z 3$} & \multicolumn{2}{|c|}{$\mathrm{m} 2-\mathrm{Z4}$} \\
\hline & $A$ & $B$ & $A$ & $\mathrm{~B}$ & $A$ & $B$ & $A$ & $B$ & $A$ & $B$ & $A$ & $B$ & $A$ & $B$ & $A$ & $B$ \\
\hline Abundancia X H & 224 & 9,72 & 17,8 & 16,9 & 34,1 & 9,56 & 31,6 & 10,2 & 634 & 55,4 & 77,3 & 28,6 & 55 & 15,2 & 85,7 & 10,6 \\
\hline Taxa X H & 13 & 2 & 1 & 5 & 3 & 2 & 2 & 3 & 15 & 9 & 4 & 3 & 4 & 5 & 4 & 2 \\
\hline Margalef X H & 2,8 & 0,72 & & 1,92 & 0,96 & 0,56 & 0,46 & 1,24 & 2,59 & 2,38 & 1,02 & 0,63 & 1,06 & 2,23 & 0,87 & 0,56 \\
\hline Abundancia X m & \multicolumn{2}{|c|}{233,5113} & \multicolumn{2}{|c|}{34,744} & \multicolumn{2}{|c|}{43,6569} & \multicolumn{2}{|c|}{41,8351} & \multicolumn{2}{|c|}{689,682} & \multicolumn{2}{|c|}{105,9773} & \multicolumn{2}{|c|}{70,205} & \multicolumn{2}{|c|}{96,2627} \\
\hline Taxa X m & \multicolumn{2}{|c|}{14} & \multicolumn{2}{|c|}{5} & \multicolumn{2}{|c|}{4} & \multicolumn{2}{|c|}{4} & \multicolumn{2}{|c|}{16} & \multicolumn{2}{|c|}{ r } & \multicolumn{2}{|c|}{7} & \multicolumn{2}{|c|}{4} \\
\hline Margalef $\mathrm{X} \mathrm{m}$ & \multicolumn{2}{|c|}{2,993} & \multicolumn{2}{|c|}{1,82} & \multicolumn{2}{|c|}{1,137} & \multicolumn{2}{|c|}{1,137} & \multicolumn{2}{|c|}{2,715} & \multicolumn{2}{|c|}{1,329} & \multicolumn{2}{|c|}{1,914} & \multicolumn{2}{|c|}{0,831} \\
\hline
\end{tabular}

\begin{tabular}{|c|c|c|c|c|c|c|c|c|c|c|c|c|c|c|c|c|}
\hline & \multicolumn{8}{|c|}{ M3 } & \multicolumn{8}{|c|}{ M4 } \\
\hline & \multicolumn{2}{|c|}{ m3-Z1 } & \multicolumn{2}{|c|}{ m3-Z2 } & \multicolumn{2}{|c|}{ m3-Z3 } & \multicolumn{2}{|c|}{ m3-Z4 } & \multicolumn{2}{|c|}{$\mathrm{m4-Z1}$} & \multicolumn{2}{|c|}{$\mathrm{m} 4-\mathrm{Z2}$} & \multicolumn{2}{|c|}{$\mathrm{m} 4-\mathrm{Z3}$} & \multicolumn{2}{|c|}{$\mathrm{m} 4-\mathrm{Z4}$} \\
\hline & $\mathrm{A}$ & $\mathrm{B}$ & A & $\mathrm{B}$ & $\mathrm{A}$ & $\mathrm{B}$ & $\mathrm{A}$ & $\mathrm{B}$ & $\mathrm{A}$ & $\mathrm{B}$ & $\mathrm{A}$ & $\mathrm{B}$ & $\mathrm{A}$ & $\mathrm{B}$ & $\mathrm{A}$ & $B$ \\
\hline Abundancia X H & 673 & 19,2 & 78,2 & 38,8 & 118 & 20,7 & 112 & 23,7 & 1877 & 108 & 270 & 59,9 & 193 & 35,7 & 269 & 21,7 \\
\hline Taxa X H & 10 & 2 & 3 & 2 & 2 & 3 & 4 & 2 & 7 & 4 & 2 & 0 & 3 & 2 & 3 & 2 \\
\hline Margalef X H & 2,1 & 1,44 & 0,72 & 0,56 & 0,37 & 0,8 & 0,79 & 0,38 & 1,94 & 1,54 & 0,91 & 0 & 1,82 & 0,72 & 1,82 & 1,44 \\
\hline Abundancia X m & \multicolumn{2}{|c|}{692,0452} & \multicolumn{2}{|c|}{116,976} & \multicolumn{2}{|c|}{138,6276} & \multicolumn{2}{|c|}{135,3404} & \multicolumn{2}{|c|}{1984,728} & \multicolumn{2}{|c|}{329,9092} & \multicolumn{2}{|c|}{228,82} & \multicolumn{2}{|c|}{291,0508} \\
\hline Taxa X m & \multicolumn{2}{|c|}{10} & \multicolumn{2}{|c|}{ 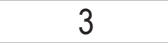 } & \multicolumn{2}{|c|}{0} & \multicolumn{2}{|c|}{1} & \multicolumn{2}{|c|}{10} & \multicolumn{2}{|c|}{2} & \multicolumn{2}{|c|}{3} & \multicolumn{2}{|c|}{4} \\
\hline Margalef $\mathrm{X} \mathrm{m}$ & \multicolumn{2}{|c|}{2,085} & \multicolumn{2}{|c|}{0,647} & \multicolumn{2}{|c|}{0,607} & \multicolumn{2}{|c|}{0,7357} & \multicolumn{2}{|c|}{2,673} & \multicolumn{2}{|c|}{0,91} & \multicolumn{2}{|c|}{1,028} & \multicolumn{2}{|c|}{1,864} \\
\hline
\end{tabular}


resultados obtenidos de diversidad son una referencia que indica que los cultivos de papa en el páramo afectan directamente el componente biológico de los suelos.

Dinámica espacial: Los organismos más representativos en el análisis de horizontes, se hallan en la zona 1, que corresponde al páramo natural en el que se encontraron 432 individuos, $62,2 \%$ del total de la población, de los cuales 390 del horizonte A y 42 al B. En las zonas 2,3 y 4 , se encontró, para los dos horizontes, un valor 263 individuos, 37,8\% del total, 170 en el horizonte A y 93 en B (Tabla 2).

Walwork (1970), Parisi (1979) y Bernal (1985) indican que la fauna se ubica en los primeros centímetros del suelo debido a que allí se presenta la mejor oferta de nutrientes por la materia orgánica, adecuados niveles de humedad y mayor disponibilidad de oxígeno que en capas más profundas. El cultivo de papa ha modificado drásticamente la distribución de la edafofauna; al comparar los horizontes A y B no se hallaron mayores diferencias. Se observó la disminución de organismos, no se encontraron abundancias superiores a 90 individuos por muestra y, de esta forma, el horizonte A de la zona cultivada en papa perdió la capacidad para sostener la abundancia y la riqueza de propia del páramo.

Similaridad: Respecto a la similaridad, empleando la riqueza, se establecieron seis grupos a un nivel de 0,85 (Figura 3). El primer grupo presentó valores medios de riqueza para la zona 1, 13 -17 morfotipos, igualmente los valores medios de riqueza 9 -11 estuvieron representados en el grupo dos que corresponde a las zonas 2, 3 y 4 . Las menores riquezas, se reportaron en las zonas 2 , 3 y 4, caso que se aprecia bien en el grupo 3; en el grupo 4, se destacó que la riqueza más baja encontrada correspondió al muestreo 4, zona 2, el cual tuvo como antecedente que ha sido trabajado sin descanso, lo que probablemente ocasionó una recuperación muy lenta. Los grupos 5 y 6 presentaron las mayores riquezas y junto con el grupo 1 marcan la diferencia del páramo frente al área empleada para el cultivo, señalando una distribución normal para la riqueza a lo largo de los meses de muestreo, condición que no se da en el área cultivada, donde las actividades agrícolas, limitan la vida de los organismos.

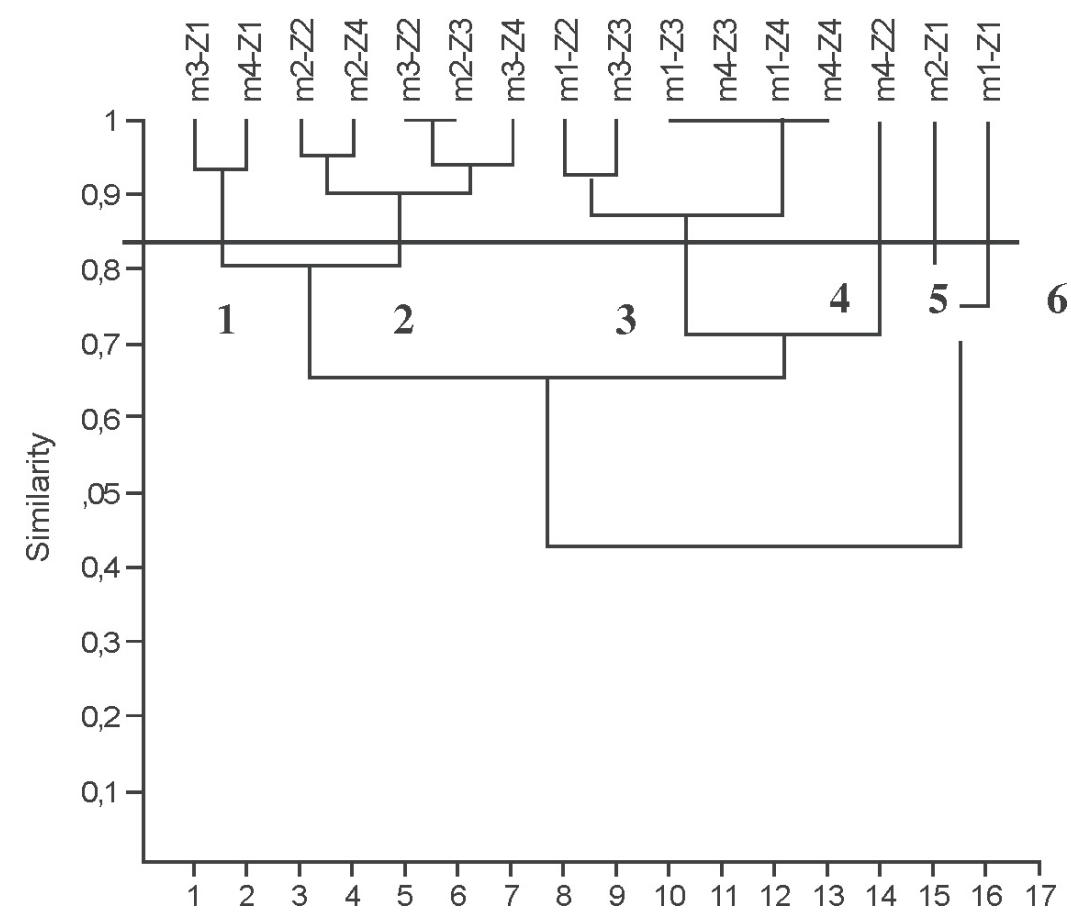

Figura 3. Dendrograma a partir de los índices de similaridad de Bray Curtis empleando la riqueza. 
Análisis de mutivariado: En la figura 4, se ve la formación de cuatro grupos: el uno, el de mayor proyección, esta integrado por los muestreos que mayor diversidad presentaron, destacándose principalmente, los muestreos 1, 2 y 4 del páramo natural; el resto estuvo integrada por las muestras de la zona agrícola.
El segundo grupo, se destacó por la diversidad media obtenida para las zonas 2 y 3 , el grupo 3 y 4 , señalaron los muestreos, con los valores de diversidad más bajos de todas las zonas. El comportamiento de las variables estuvo directamente relacionado y confirmaron los resultados obtenidos a lo largo de este estudio.

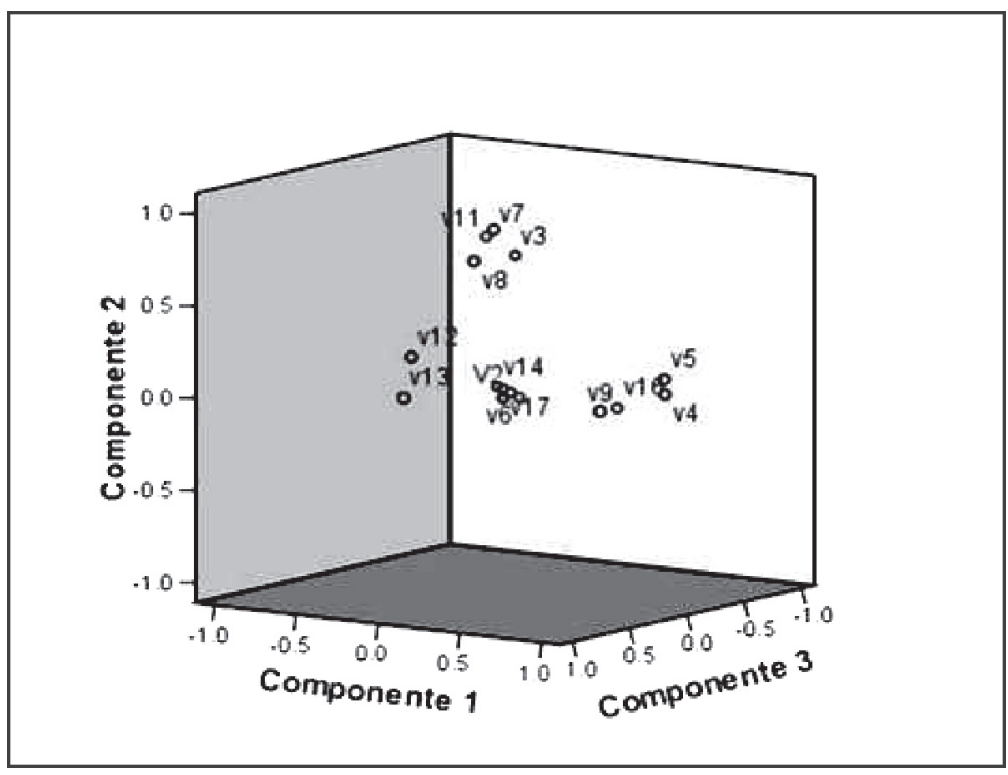

Figura 4. Componentes principales con base en la diversidad de las zonas V2-V5 = Muestreo 1 zonas 1-2-3-4, V6-V9 muestreo 2 zonas 1-2-3-4, V10-V13 =Muestreo 3 zonas 1-2-3-4, V14-V17 Muestreo 4 zonas1-2-3-4.

\section{CONCLUSIONES}

La fauna edáfica del páramo pose una alta especificidad de hábitat y recursos para el desarrollar su ciclo de vida, el laboreo del suelo repercutió en la pérdida de grupos taxonómicos disminuyendo su frecuencia, su constancia y su dominancia en el tiempo; de esta forma, la regeneración de las poblaciones del suelo queda supeditada a las oportunidades que pueda generar el hombre en sus actividades.

El establecimiento de cultivos en el páramo ocasiona cambios en la composición y en la abundancia de la edafofauna. Algunos grupos desaparecen mientras que otros, como las especies cosmopolitas, se incrementan o adaptan a las condiciones del medio, como es el caso de Acari, larvas de Coleoptera, Entomobryidae, Psocoptera, Aphidoidae y Staphylinidae.

El cultivo de papa modifica drásticamente la distribución de la edafofauna; al comparar los horizontes A y B no se hallan mayores diferencias. El horizonte A de la zona cultivada en papa pierde la capacidad para sostener la abundancia y la riqueza de fauna edáfica propia del páramo.

En general, el páramo natural proporciona todas las condiciones para que la fauna edáfica se mantenga con sus características, composición y dinámica. El cultivo de papa, independientemente del estado en que se encuentre, altera de forma drástica la estructura ecológica de las comunidades de la fauna edáfica, disminuyendo su composición, su abundancia y su riqueza.

\section{AGRADECIMIENTOS}

Los autores agradecen a la Universidad de Ciencias Aplicadas y Ambientales, U.D.C.A y COLCIENCIAS por el apoyo al Grupo Páramo para la realización de la investigación a través de la financiación del programa Jóvenes Investigadores e Innovadores. 


\section{BIBLIOGRAFÍA}

ALCALDÍA MUINICIPAL CARMEN DE CARUPA, 2007. Plan de Desarrollo Municipal, 2004-2007. 420p.

ALARCÓN, H.; BARBOSA, C.C.; ARGUELLO, S.; RAMIREZ, A.; SALAZAR, H.F.; VILLE, T.J.; VILLA, L.A. 2002. Transformaciones y cambio en el uso del suelo en los Paramos de Colombia en las ultimas décadas. En: IDEAM; MMA; NUD Eds. Páramos y Ecosistemas Alto Andinos en Condiciones Hot Spot E Global Climática Tensor. p.211-333.

ANDERSON, J.M.; INGRAM, J.S. 1989. Tropical Soil Biology and Fertility. A Handbook of Methods. CAB International. Walligford, UK. p. 59-69.

ANDRADE, G.; ROSAS, M L.; ARANGO, F.G. 2002. Lineamientos para definir la vulnerabilidad y adaptabilidad de los ecosistemas continentales colombianos ante la convención de cambio climático. En: IDEAM; MMA; NUD Eds. Páramos y Ecosistemas Alto Andinos en Condiciones Hot spot E Global Climática Tensor. p.71-160.

AVELLANEDA, A. 1998. Limitaciones en la aplicación de la normativa ambiental para la gestión en páramos. En: Memorias Curso Caracterización y Manejo de la Zonas de Páramo. MMA, ICFES, BID. Villa de Leyva. 185p.

BERNAL, C.A. 1985. Estudio comparativo de la entomofauna del pajonal paramuno y del bosque alto andino de la región de Monserrate Cundinamarca. Ecología de los Paramos Andinos, Una visión preliminar integrada. Inst. Ciencias Naturales. Biblioteca José Jerónimo Triana No 9. p. 225-260.

BORROR, D.J.; TRIPLEHORN, C.A. 1989. An introduction to the study of insects. $6^{\mathrm{a}} \mathrm{Ed}$. Saunders College Publ. Philadelphia. EEUU. 875p.

CASTAÑO, U.; FRANCO, V. L.; REY, C. 2003 . El páramo en Colombia. Hoftede, R; Segera, P; MENA, P. Ed. Los Páramos del Mundo. 59p.

CORTÉS, L.A. 1995. Los suelos de páramo: Regulador del recurso hídrico en Boyacá. En: Fundación de
Ecosistemas Altoandinos Ed. El Páramo. Ecosistema de Alta Montaña. Serie Montañas Tropoandinas. Fundación de Ecosistemas Andinos. Bogotá. 1:85-93.

ESTUPIÑAN, L.H. 2001. Los páramos, ecosistemas para conservar. Rev. U.D.C.A Actualidad \& Divulgación Científica. 4(1):7-12.

GÓMEZ, S.C. 2002. Cambios y transformaciones en el suelo del bioma del páramo por el cambio climático. Memorias Congreso Mundial de Páramos Tomo I. Paipa (Colombia). p.72-79.

GONZÁLES, F.; CÁRDENAS, F. 1995. El páramo un paisaje deshumanizado: El caso de las provincias del Norte y Gutiérrez (Boyacá-Colombia). En: Reyes, P., Molano, J. Eds. El páramo, Ecosistema de Alta Montaña. Serie Montañas Tropoandina, Vol. 1. Fundación Ecosistemas Andinos. Editorial Fundación Cultural Javeriana de Artes Gráficas. Bogotá. p. 25 - 44.

GUHL, E. 1982. Los paramos que circundan la Sabana de Bogotá. Jardín Botánico "José Celestino Mutis" Bogotá. 120p.

HOFFMANN, A. 1996. Animales desconocidos relatos acarológicos. Fondo de Cultura de Económica. México. 215p. Disponible desde Internet en: (con acceso 12/10/06).

HOFSTEDE, R. 2003. Los Páramos en el Mundo: su diversidad y sus habitantes. En: Hofstede, R.; Segara, P.; Mena, P. Eds. Los Páramos del Mundo. p.15-38.

INFANTE, J. 1987. Influencia del uso del suelo sobre la mesofauna edáfica en el Páramo de Chingaza, Cundinamarca Colombia. Tesis de grado. Fac. Ciencias, Depto. Biología. U. N. de Colombia. 240p.

MALAGÓN, D.; PULIDO, C. 2000. Suelos del Páramo colombiano. En: Rangel, J.O. (Ed). Colombia Diversidad Biótica III. La Región de la vida Paramuna. U. N. de Colombia. p.37-84.

MARÍN, P.E.; FEIJOO, A.; PEÑA, J.J. 2001. Cuantificación de la macrofauna en un vertisol bajo dife- 
rentes sistemas de manejo en el Valle del Cauca Colombia. Suelo Ecuatoriales. 31(2):233-238.

MORALES, J.; SARMIENTO, L. 2002. Dinámica de los macroinvertebrados edáficos y su relación con la vegetación en una sucesión secundaria en el Páramo Venezolano. Ecotrópicos. 15(1):99-110.

PARISI, V. 1979. Biología y ecología del suelo. Edit. Blume. España. 169p.

PASHANASI, B. 2001. Estudio cuantitativo de la macrofauna del suelo en diferentes sistemas de uso de la tierra en la Amazonia Peruana. Folia Amazónica. 12(1-2):75-97.

PEREDO, P.S.; BARRERA, C.P.; PARADA, Z.E. 2002. Efecto de prácticas agrícolas convencionales sobre la biodiversidad de los grupos mesofaunísticos edáficos en un huerto de ciruelos. Una aproximación agroecológica. Agro Sur. 30(2):7-14.

PETERSON, A. 1960. Larvae of insects. Ed. Edward's Brothers. Inc-Ann Arbor. Michigan. 416p.

PRIETO, P.J.; CASASBUENAS, P.L . 2005. Impacto de la Quema sobre la fauna edáfica en el Páramo Rabanal, Samacá Boyacá. Ingeniería Agronómica, Universidad de Ciencias Aplicadas y Ambientales U.D.C.A. Bogotá. 86p.

RAMÍREZ, R.P.; TRUJILLO, R.P.; RIVERA, C.B. 2005. Identificación y cuantificación de la actividad microbiana y macro fauna de un andisol bajo diferentes sistemas de manejo, en el municipio de Marinilla Antioquia. Universidad Nacional. Medellin. p.1-26.

RUIZ, H.D. 2006. Efectos de diferentes grados de perturbación del ecosistema sobre la macrofauna del suelo. Depto. Biología U. La Habana. Disponible desde Internet en: http:/humboldt.org.co/chmcolombia/servicios/jsp. (con acceso 20/06/06).

SALAMANCA, P.N.; CHAMORRO B.C. 1994. La edafofauna del páramo de Monserrate - Hacienda "Santa Bárbara"- (Cundinamarca - Colombia). En: Mora-Osejo, L.; Sturm, H. Eds. Estudios ecológicos del páramo y del bosque altoadino. Cordillera Oriental de Colombia. Tomo II. Acad. Col. de Cienc. Exactas, Físicas y Naturales; Col. Jorge Álvarez Lleras No. 6:631-647.

VANEGAS, O.V. 2002. Influencia de algunas características del hábitat sobre la edafofauna de un bosque altoandino y una plantación de pino. Tesis de Grado: Depto. Biología. U.N. de Colombia. Bogotá. 70p.

VARELA, R. A. 2006 Grupos funcionales microbianos y servicios ecosistémicos del suelo. Memorias Congreso del Suelos 2006.

VARGAS-RÍOS, O.; RIVERA, D. 1990. El páramo un ecosistema frágil. Cuadernos de Agroindustria y Economía Rural. No. 25. Pontificia Universidad Javeriana. Bogotá. 25p.

WALLTER, D.E.; PROCTOR , H.C. 2005. Orders, suborders $E$ cohorts of mites in soil. Disponible desde Internet en: http:www//lucidcentral.com/keys/cpitt/ public/Mites/Soil\%20mites/index.htm (con acceso 15/07/06)

WALWORK, J. 1970. Ecology of Soil Animals, Mc Graw Hill. London. 283p.

Recibido: marzo 21 de 2007

Aceptado: septiembre 14 de 2007 